\title{
ED AT YOUNG AGE PREDICTS HEART DISEASE
}

\section{RISK FACTORS}

Young men who develop erectile dysfunction (ED) are at increased risk for future coronary artery disease (CAD), according to researchers from the Mayo Clinic. Studies have previously linked ED with an increased incidence of heart disease, but a relationship with age had not been investigated. "We found that the highest risk of new heart disease was in the youngest study participants with ED," says Jennifer St Sauver, corresponding author on the paper. "ED may be an important predictor of coronary heart disease among young men."

The researchers investigated a cohort of white men who participated in a long-term county-wide study of urologic health in the US. In total, 1,402 men aged $>40$ years who had a regular sexual partner and no evidence of CAD at baseline were included in the study. Participants completed the Brief Male Sexual Function Inventory (BMSFI; a validated self-report questionnaire) at 2-year intervals during the decade from 1996. The researchers assessed cardiovascular outcomes by extracting data from a concurrent long-term study of heart disease among county residents.

Men with ED were $80 \%$ more likely to develop incident CAD than were those without ED, after adjustment for cardiovascular risk factors. The association between ED and CAD seemed strongest in men aged 40-49 years, and diminished with increasing age. The authors conclude that ED in younger men is associated with a marked increase in the likelihood of future CAD; ED in older men, by contrast, is of little prognostic importance.

Both ED and CAD have been suggested to be manifestations of a common underlying vascular pathology. ED seems to precede heart disease by a number of years, which raises the possibility of a 'window of curability' for younger men, during which intervention could delay or prevent the emergence of overt cardiac disease. Young men with ED might, therefore, be good candidates for trials of cardiovascular disease prevention.

"Further studies designed to determine what it is about these young men that might predispose them to an increased risk of heart disease would shed light on whether specific interventions might decrease this risk," suggests St Sauver. "Results from such studies could then help in designing clinical trials to determine whether such interventions might truly be effective in preventing morbidity and mortality from heart disease later in life."

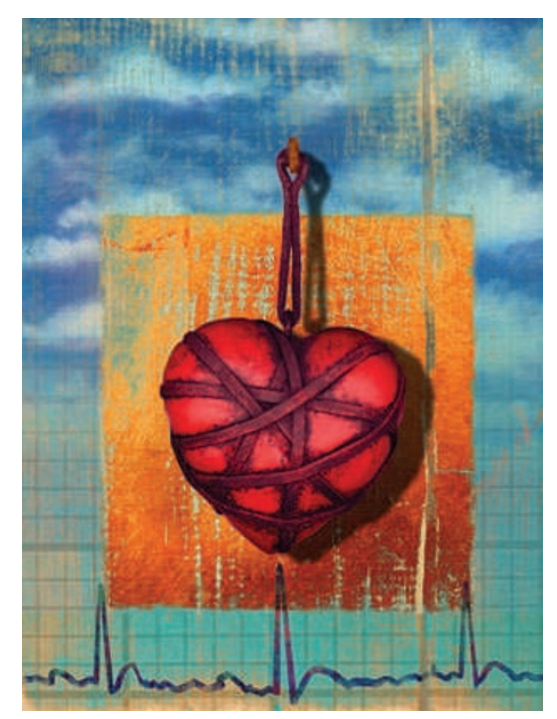

\section{Nick Groves-Kirkby}

Original article Inman, B. A. et al. A population-based, longitudinal study of erectile dysfunction and future coronary artery disease. Mayo Clin. Proc. 84, 108-113 (2009). 